\title{
$\mathrm{M}|\mathrm{R}| \mathrm{S}$ Internet Journal Nitride Semiconductor Research
}

\section{The effects of indium concentration and well-thickness on the mechanisms of radiative recombination in $\operatorname{In}_{x} \mathrm{Ga}_{1-\mathrm{x}} \mathrm{N}$ quantum wells}

\author{
N. A. Shapiro ${ }^{12}$, Piotr Perlin ${ }^{123}$, Christian Kisielowski ${ }^{4}$, L. S. Mattos ${ }^{12}$, J. W. Yang ${ }^{5}$ and Eicke R. \\ Weber ${ }^{12}$ \\ ${ }^{1}$ Materials Science Division, Lawrence Berkeley National Laboratory, Berkeley CA 94720 , \\ ${ }^{2}$ University of California at Berkeley, Department of Materials Science and Mineral Engineering, \\ ${ }^{3}$ High Pressure Research Center, \\ ${ }^{4}$ NCEM, Lawrence Berkeley Laboratory, \\ ${ }^{5}$ APA Optics Inc.,
}

(Received Monday, December 13, 1999; accepted Tuesday, February 1, 2000)

\begin{abstract}
A correlation of the local indium concentration measured on an atomic scale with luminescence properties of $\operatorname{In}_{\mathrm{x}} \mathrm{Ga}_{1-\mathrm{x}} \mathrm{N}$ quantum wells reveals two different types of recombination mechanisms. A piezoelectric-field based mechanism is shown to dominate in samples with thick wells $(\mathrm{L}>3 \mathrm{~nm}$ ) of low indium concentration $(x<0.15-0.20)$. Spatial indium concentration fluctuations dominate luminescence properties in samples of higher indium concentrations in thinner wells. Quantum confinement is shown to have a major effect on the radiative recombination energy. A model is presented that relates the experimentally measured nano scale structural and chemical properties of quantum wells to the characteristics of the luminescence.
\end{abstract}

\section{Introduction}

$\mathrm{In}_{\mathrm{x}} \mathrm{Ga}_{1-\mathrm{x}} \mathrm{N}$ has emerged in the last years as the most important material for short-wavelength optoelectronics. A successful application of this material in the active layers of light-emitting diodes (LED) and laser diodes (LD) [1] convincingly demonstrates the enormous potential of the group III nitrides. However, even though devices based on this material are already available in electronics stores, the nature of the radiative transitions that occur in these devices is still under debate [2]. Over the past few years, the literature has witnessed reports of observations and arguments for two different radiative recombination mechanisms. One is based on spatial indium fluctuations [3] [4] [5] [6] [7] [8] [9] [10] [11] [12] and the other on piezoelectric fields in the layers [13] [14] [15]. In this report, we show that in fact both mechanisms may operate in these devices, and that the engineering of the device can determine which of the two will dominate its luminescence behavior.

In the first model, the indium concentration in quantum wells is assumed to fluctuate spatially, thus forming deep cusps or "quantum dots" in the energy gap [12].
Exciton pairs are confined in the local minima, and the cusps operate as excellent radiative recombination centers. Quantitative high-resolution transmission electron microscopy (HRTEM) proved the presence of spatial indium fluctuations [11] [16]. The second model attributes the quantum-confined Stark effect (QCSE) to the presence of a large piezoelectric field in the well [14]. This piezoelectric field arises from strain that is caused by the lattice mismatch between $\mathrm{GaN}$ and $\mathrm{In}_{\mathrm{X}} \mathrm{Ga}_{1-\mathrm{x}} \mathrm{N}$. According with the QCSE model, the field causes the energy bands to "bend," thereby inducing charge separation and a "red" shift of the emission. The magnitude of shift is related to the strength of the field and to the thickness of the layer across which it operates.

This report presents quantitative HRTEM data together with photo- and electroluminescence measurements that determine which of these two recombination mechanisms dominates. It is found that the average indium concentration and the well thickness are the critical parameters that determine which recombination mechanism will dominate in the layer. 


\section{Experiments}

Four samples grown by Metal Organic Chemical Vapor Deposition (MOCVD) were studied: commercially available green (sample 1) and blue (sample 2) LEDs manufactured by Nichia Chemicals, a multi-quantum well sample manufactured by APA Optics (sample 3), and another multi-quantum well sample (sample 4). Quantitative structural and chemical information was extracted by a combination of Secondary Ions Mass Spectrometry (SIMS), Rutherford Back Scattering (RBS), Electron Energy Loss Spectroscopy (EELS) and HRTEM [11] [15]. The active region of sample 1 consists of a $2.5-\mathrm{nm}$ thick $\operatorname{In}_{\mathrm{x}} \mathrm{Ga}_{1-\mathrm{x}} \mathrm{N}$ layer $(\mathrm{x}=0.22 \pm 0.04)$, where $\mathrm{x}$ is the indium fraction on the Group III lattice sites. Sample 2 has the same indium content, but the quantum well thickness is $1.5 \mathrm{~nm}$. The APA structure consists of a $1.85-\mu \mathrm{m}$ thick $\mathrm{GaN}$ layer followed by a series of six quantum wells. Each quantum well consists of an approximately 5-nm thick $\operatorname{In}_{\mathrm{x}} \mathrm{Ga}_{1-\mathrm{x}} \mathrm{N}$ layer sandwiched between two 20 -nm thick GaN barriers and is repeated twice. The average $\mathrm{x}$ values are $0.06,0.1$ and 0.15 , for the first, second and third pairs of wells, respectively. The thickness of these wells is $3.5 \mathrm{~nm}, 4.5$ $\mathrm{nm}$ and $5.3 \mathrm{~nm}$, respectively. Sample 4 consists of a series of ten 1.5-1.7 nm thick quantum wells with identical $x$ values of $0.32 \pm 0.08$.

Photo- and electroluminescence spectra were recorded using a $0.85-\mathrm{m}$ double monochromator with a GaAs photomultiplier. A 325-nm He-Cd laser was used to excite the photoluminescence. An incident power of roughly 5-25 $\mathrm{mW}$ was focused on a spot of $10-\mu \mathrm{m}$ diameter. The commercial Nichia LEDs were de-encapsulated, and fixed directly to the cryostat coldfinger, and then rewired. This ensured that the temperature measured on the cold finger was the same as that of the diode.

For HRTEM, the cross section samples were mechanically thinned and ion milled to electron transparency. A short etching procedure removed the surface damage produced by the milling process [11]. The Atomic Resolution Microscope was operated at $800 \mathrm{kV}$ to produce lattice images. The digitized lattice images were exploited to measure local strain profiles using the following procedure. A search program determines the position of each individual "blob" in images with sub pixel accuracy. This position relates to atomic columns in the sample and can be used to measure the size of each structural unit cell. From these measurements, the a- and c-lattice parameters are determined on an atomic scale. The local strain is defined as the deviation of the local lattice parameter from that of the $\mathrm{GaN}$ matrix. Since the InGaN layer is epitaxially grown on $\mathrm{GaN}$, the $a$-lattice parameter of the layer is constrained to equal that of the GaN matrix. The $c$-lattice parameter on the other hand, expands or contracts depending on the sign of the stresses. A calibration procedure and Vegard's law were used to relate this strain to local alloy concentration [11]. Strain relaxation processes were minimized by producing images from areas that were 10-50 times thicker than the width of the wells. The electron acceleration voltage of $800 \mathrm{kV}$ in the Atomic Resolution Microscope was utilized for this purpose. Such methods were developed for strain profiling and mapping in cubic and hexagonal systems [17] [18].

\section{Results}

Figure 1 summarizes essentials of the HRTEM analysis [11]. It depicts strain profiles across quantum wells recorded at a spatial resolution of $0.5 \mathrm{~nm}$. These profiles were obtained by averaging local values of $c$-strain across an image. As previously discussed, these strain profiles correspond directly to the indium concentration profiles, which are also included in Figure 1. Thus, Figure 1 shows the shapes (thickness and depth) of the quantum wells in samples 1 through 4 . The Figure shows that the quantum well in sample 3 is significantly broader $(\sim 5 \mathrm{~nm})$ and of lower indium concentration compared with the other samples. Sample 4 shows the highest indium concentration and a well thickness that is similar to that of the blue Nichia LED (sample 2, 1.5 $\mathrm{nm}$ ). The indium concentrations in the blue and the green Nichia LEDs (samples $1 \& 2$ ) are similar, but the well width in sample 1 is significantly broader $(\sim 2.5$ $\mathrm{nm})$. The inset depicts that in all cases blue and green emission was observed that does NOT scale with the indium concentration.

While Figure 1 shows the local average strain in the well, Figure 2 shows its standard deviation. The standard deviation of the $c$-strain is interpreted in terms of indium composition fluctuation in the well. The indium fluctuation calculation is based on the previously made correlation between the indium fraction and $c$-strain. Figure 2 shows that as the average indium fraction increases, the indium composition fluctuations also increase.

Photoluminescence experiments of samples 1 through 4 were done for a range of excitation power densities at $300 \mathrm{~K}$. The photoluminescence peak energies are shown as a function the of excitation power in Figure 3. Significant results are pointed out next. First, the energy of the luminescence peak of sample 3 is considerably more sensitive to the excitation power than that of any of the other samples. Second, the peak energies of samples 1,2 and 4 do not correlate with the indium content at any excitation level. Samples 1 and 2, which have similar indium concentrations, exhibit luminescence at greatly differing energies, and sample 4, 
which has a greater indium content than sample 1, exhibit luminescence at a higher energy than sample 1.

The electroluminescence peak energy of sample 1 is shown in Figure 4 as a function of the current for 50, 177 and $295 \mathrm{~K}$. A pattern is seen in the temperature-current dependencies of the peak. Note that at low currents, the peak energy increases with increasing temperature even though the energy gap should be shrinking. The following simulation shows that this pattern is consistent with a recombination mechanism based on spatial fluctuations of the energy gap in the layer.

\section{Band-Tails Simulation}

Eliseev et al. [9] recently proposed that the radiative recombination in Nichia devices could be described as a transition between the density of states tail of the valence and conduction bands. Such band tails are the natural result of spatial fluctuations in the energy gap. In the case of a layer dominated by quantum dots, these tails are the result of fluctuation both in the indium composition of the dots and the size of the dots. Eliseev's model was used to explain the anomalous temperature dependence of the emission from Nichia LEDs. We now extend this model to explain both the temperature and the power induced shifts of the emission. The model assumes that the density of states (DOS) distribution of the tails can be described by the Gaussian functions

$$
\rho_{e, h}=\beta_{0, h} \exp \left[\frac{-\left(E-E_{0 e, h}\right)^{2}}{2 \sigma_{e, h}^{2}}\right],
$$

where $\rho_{0 \mathrm{e}}, \rho_{0 \mathrm{~h}}, E_{0 \mathrm{e}}, E_{0 \mathrm{~h}}, \sigma_{0 \mathrm{e}}$ and $\sigma_{0 \mathrm{~h}}$ are fixed parameters for band-tails of electron and hole states. $\rho_{0 \mathrm{e}}$ and $\rho_{0 \mathrm{~h}}$ are the magnitudes for the normal densities of state for the ground state of a quantum well for the conduction and valence bands, respectively. They are can be calculated by

$$
\rho_{0 e, h}=\frac{m_{e, h}^{*}}{n^{2} d}
$$

where $m^{*}, h$ are the electron and hole effective mass, and $d$ is the thickness of the quantum well. $E_{0 \mathrm{e}}$ and $E_{0 \mathrm{~h}}$, correspond to the normal energy gap edges, while $\sigma_{0 \mathrm{e}}$ and $\sigma_{0 \mathrm{~h}}$ are the parameters that describe the width of the band tails. Since $E_{0 \mathrm{e}}$ and $E_{0 \mathrm{~h}}$ correspond to the band edges of the layer, the distance between them will vary with the band gap. Therefore,

$$
E_{0 e}-E_{0 h} \cong E_{g}(T)=E_{r}-\frac{\gamma}{\beta+T} \cdot T^{2}
$$

where $\gamma$ is $9.4 * 10^{-4} \mathrm{eV} / \mathrm{K}$ and $\beta$ is $770 \mathrm{~K}$ [19] and $E_{r}$ is the distance between the band edges at $0 \mathrm{~K}$. Since $E_{r}$ is very difficult to calculate accurately due to uncertainty in the bowing parameter and confinement energy, it is used as a fitting parameter in this simulation. Using $E_{r}$ as a fitting parameter does not weaken the results of this simulation because the simulation attempts to reproduce only the effects of temperature and excitation levels on the relative position of the peak energy, not its absolute value. $E_{r}$ was set so that the simulated peak position at $295 \mathrm{~K}$ and low excitation levels would match with the experimental peak position at $295 \mathrm{~K}$ and low EL currents.

The band tails model also assumes that the tail states are localized enough to relax the momentum conservation rule. In this situation, an emission spectrum can be represented by the equation:

$$
R_{s p}(h d)=\int \rho_{e}(E+h d) f_{e}(E+h d) A_{h}(E) f_{h}(E) d E,(4)
$$

where $f_{\mathrm{e}}(E+h v)$ and $f_{\mathrm{h}}(E)$ are the Fermi occupation functions for the two involved tails, each being characterized by a quasi-Fermi level. The magnitude of $R_{s p}(h v)$ corresponds to the likelihood that a radiative recombination event resulting in a photon of an energy $h v$ will occur. The integral was evaluated numerically for a spectrum of $h v$ at given temperature and hole- and electron-quasi-Fermi energy levels that correspond to specific injected carrier concentrations. A Gaussian fit of each resulting set of calculations yielded the peak energy and a linewidth of the simulated spectrum. A background carrier concentration of $10^{18} \mathrm{~cm}^{-3}$ donors had to be assumed in order to reproduce the experimental results best. It may stem from the heavy $\mathrm{n}$ doping of the device. The tail parameters $\sigma_{0 \mathrm{e}}$ and $\sigma_{0 \mathrm{~h}}$ were adjusted to obtain the right value of the emission peak linewidth for low excitation powers at room temperature. $49+/-1 \mathrm{meV}$ proved to be the best value for both parameters.

The results of the simulation are included in Figure 4. It is evident that the band-tail-recombination model describes closely the basic features of the luminescence properties of sample 1. It reproduces the anomalous temperature dependence of the emission peak energies in the low current region and the crossover at higher currents. 


\section{Discussion}

Next, the results presented in Figure 3 are explained. The large "blue" shift of sample 3 with increasing excitation power agrees with predictions of the piezoelectric model [15] [13]. The absence of this shift in samples 1 , 2 and 4 should be explained. One explanation stems from the observation that sample 3 has the thickest well out of the 4 samples. The thicker the sample well is, the greater is the spatial charge separation and potential drop across the well. This means that the QCSE will be stronger in such a structure. Consequently, the excitation induced "blue shift" is expected to be stronger in structures with thicker wells. However, rough calculations show that a $2.5-\mathrm{nm}$ thick quantum well with $\mathrm{x}=0.22$ (sample 1) should still exhibit a piezoelectric-field induced potential drop of about $70 \%$ as compared with that of a $5.3 \mathrm{~nm}$ broad well with $\mathrm{x}=0.15$ (sample 3). Thus, we believe that an additional mechanism reduces the effect of the QCSE mechanism. A quantum well with large spatial indium fluctuations will not have a smooth, sloping band structure that is necessary for the QCSE. The carriers will reside in local minima created by the fluctuations instead of separating to opposite sides of the well. Thus, the QCSE will be reduced or even eliminated in wells with large indium fluctuations.

This explanation fits with the evidence presented in this report. Samples 1, 2 and 4 have a higher average indium concentration than sample 3, and according to Figure 2, the indium spatial fluctuation increases with increasing average indium concentration [11]. Thus, sample 3, which has the lowest indium content, is dominated by the piezoelectric-field effect, while the rest of the samples are not. Also, it is noteworthy that most literature data show piezoelectric-effect dominated luminescence properties most convincingly for $\mathrm{x}$ values no greater than 0.15 [14] [13]. An exception to this rule, reported by Mukai et al. [20], was a quantum well with an indium content well over 0.15-0.20 and a thickness of $6 \mathrm{~nm}$. It is possible that the potential drop in this structure was so large (due to large strain and thickness of the well) as to overcome the potential fluctuations due to the spatial indium fluctuations.

The key to understand the order in energy of the luminescence peaks of the 4 samples is to consider quantum confinement, which has been shown to dominate in quantum wells of comparable thicknesses [4] [14] [13]. Though samples 1 and 2 have the same indium concentration, sample 2 has a smaller thickness and thus greater confinement energy. Therefore it emits at a higher energy than sample 1 . Similarly, sample 4 experiences greater confinement energy than sample 1 and therefore emits at a higher energy, even though it has a higher indium concentration.
At first glance it is difficult to see how the recombination mechanism can depend simultaneously on indium fluctuations and on the thickness of the layer. The dependence on indium composition fluctuations implies that the carrier confinement depends on the size of the quantum dot. On the other hand, the dependence on the thickness of the InGaN layer implies that the carrier confinement depends on the thickness of the entire InGaN layer. This apparent contradiction is solved when we conclude that the diameter of the quantum dot is directly proportional to the thickness of the layer. This conclusion is based on direct HRTEM observation of the dots inside the layer where the dots are shown to have a diameter that is roughly equal to the thickness of the layer [5] [8].

There are several advantages to having a device dominated by the indium fluctuation mechanism. First, the presence of spatial indium fluctuations in the layer improves the radiative recombination efficiency due to an increased localization of the excitons [10]. In addition, at the recombination centers, the indium concentration is higher than the average indium concentration in the layer. Therefore, the resulting radiation is at lower energies than that expected based on the average indium concentration. Finally, the energy of recombination or "color" of the emission is less sensitive to the excitation power or "brightness" of the device than for a device based on the piezoelectric field mechanism. We suspect that the presence of the indium fluctuations in the investigated devices is what gives them their superior luminescence properties.

\section{Conclusions}

We conclude that both the piezoelectric-field effect and spatial indium fluctuations must be taken into account to understand the radiative recombination processes in $\mathrm{In}_{\mathrm{x}} \mathrm{Ga}_{1-\mathrm{x}} \mathrm{N}$ quantum wells. Piezoelectric-field effects are likely to dominate in relatively thick wells $(\mathrm{L}>3 \mathrm{~nm})$ of low indium concentrations $(x<0.15-20)$. Indium composition fluctuation effects are likely to dominate in structures where the average indium concentration is high due to an increase in the magnitude of fluctuations. The excitation power dependence of the luminescence is indicative of the recombination mechanism that dominates in a given structure. The anomalous temperature dependence that has been observed in some structures can be explained through the use of the bandtails model that is based on spatial indium fluctuations. Quantum confinement effects cause significant shifts of the emission wavelength if the thickness of the wells is smaller than $3 \mathrm{~nm}$, which is typically the case in the investigated device structures. 


\section{ACKNOWLEDGMENTS}

The authors would like to thank J. Krueger and P. McEuen for stimulating discussions. This work was supported by the Director, Office of Energy Research, Office of Basic Energy Sciences, Materials Sciences Division, of the US Department of Energy under Contract No. DE-AC03-76SF00098.

\section{REFERENCES}

[1] Shuji Nakamura, Gerhard Fasol, The Blue Laser Diode - GaN based Light Emitters and Lasers , (Springer-Verlag, Heidelberg, 1997),

[2] S. J. Pearton, J. C. Zolper, R. J. Shul, F. Ren, J. Appl. Phys. 86, 1 (1998).

[3] Y. Narukawa, Y. Kawakami, S. Fujita, S. Fujita, S. Nakamura, Phys. Rev. B 55, R1938 (1997).

[4] S. Chichibu, T. Azuhata, T. Sota, S. Nakamura, Appl. Phys. Lett. 69, 4188-4190 (1996).

[5] Y. Narukawa, Y. Kawakami, M. Funato, S. Fujita, S. Fujita, S. Nakamura, Appl. Phys. Lett. 70, 981-983 (1997).

[6] S Chichibu, T Azuhata, T Sata, S Nakamura, Appl. Phys. Lett. 70, 2822-2824 (1997).

[7] Shigefusa Chichibu, Kazumi Wada, Shuji Nakamura, Appl. Phys. Lett. 71, 2346-2348 (1997).

[8] C. Kisielowski, Z. Liliental-Weber, S. Nakamura, Jpn. J. Appl. Phys. 36, 6932 (1997).

[9] PG Eliseev, P Perlin, J Lee, M Osinski, Appl. Phys. Lett. 71, 569 (1997).

[10] T. Mukai, M. Yamada, S. Nakamura, Jpn. J. Appl. Phys. 37, 1358 (1998).

[11] C. Kisielowski, "Composition and Strain Fluctuations in InN/GaN/AlN Heterostructures: A Microscopic Glimpse below Surfaces", Proceedings of the 2nd International Symposium of blue Laser and Light Emitting Diodes, 321 (Ohmsha Ltd., 1998)

[12] K. P. O'Donnell, R. W. Martin, P. G. Middleton, Phys. Rev. Lett. 82, 237 (1999).

[13] T. Takeuchi, S. Sota, M. Katsuragawa, M. Komori, H. Takeuchi, H. Amano, I. Akasaki, Jpn. J. Appl. Phys. 36, 382 (1997).

[14] Andreas Hangleiter, Jin Seo Im, H. Kollmer, S. Heppel, J. Off, Ferdinand Scholz, MRS Internet J. Nitride Semicond. Res. 3, 15 (1998).

[15] P. Perlin, C. Kisielowski, V. Iota, B. Weinstein, L. Mattos, N.A. Shapiro, J. Kruger, E.R. Weber, J. Yang, Appl. Phys. Lett. 73, 2778-2780 (1998).

[16] C. Kisielowski, 57, 306 (1999).

[17] H. Seitz, M. Seibt, F. H. Baumann, K. Ahlborn, others, Phys. Stat. Sol. A 150, 625 (1995).

[18] C. Kisielowski, O. Schmidt, J. Yang, Mater. Res. Soc. Symp. Proc. 482, 369 (1998).

[19] H. Teisseyre, P. Perlin, T. Suski, I. Grzegory, S. Porowski, J. Jun, A. Pietraszko, T. D. Moustakas, J. Appl. Phys. 76, 2429-2434 (1994).

[20] T. Mukai, M. Yamada, S. Nakamura, Jpn. J. Appl. Phys. 38, 3976 (1999).

\section{FIGURES}

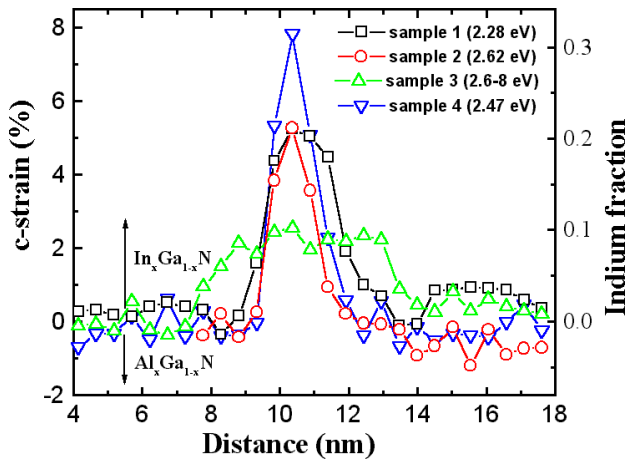

Figure 1. Average strain profiles across the active region of samples 1 through 4. Also shown is the indium fraction that corresponds to the $c$-strain. Light emission energies are indicated [8].

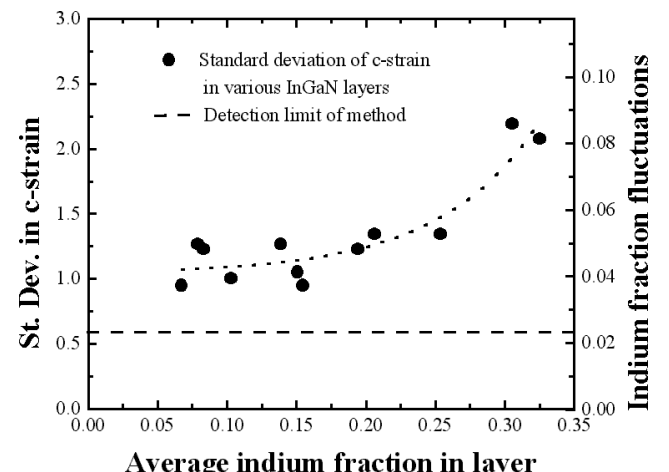

Figure 2. Standard deviation of the c-strain in InGaN layers with various indium fractions. Also shown are the indium fraction fluctuations that correspond to the c-strain fluctuations. The detection limit is the standard deviation of the c-strain in the surrounding GaN matrix.

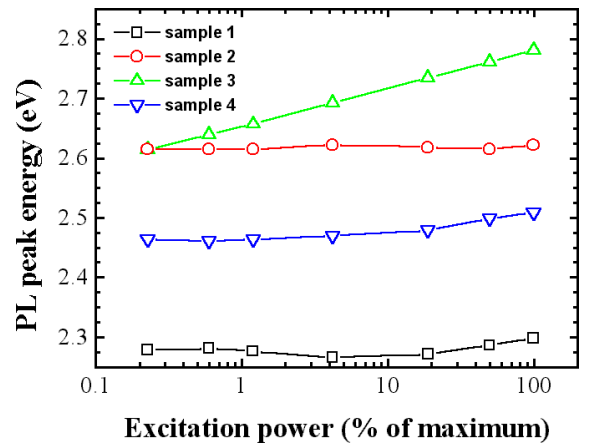

Figure 3. Energies of photoluminescence (PL) peaks of samples 1 through 4 are plotted as a function of excitation power at $300 \mathrm{~K}$. 


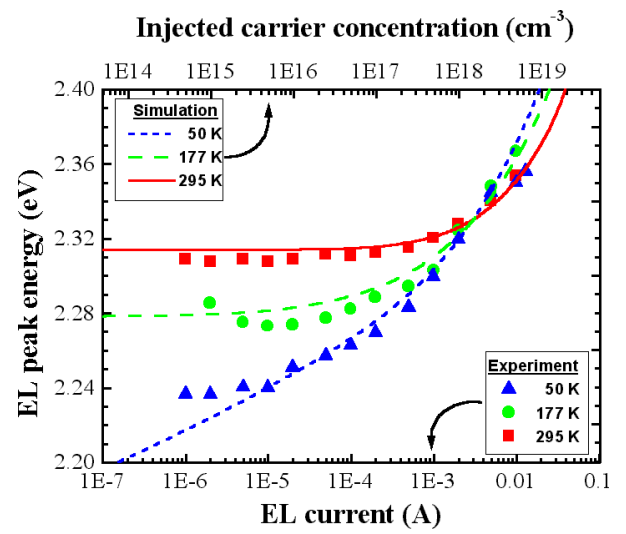

Figure 4. Energy of electroluminescence (EL) peak for a Nichia green LED (sample 1) as a function of temperature and current. The lines show the results of calculations using the Bandtails model. 